\title{
SENSIBILIZAÇÃO DA BLOMIA TROPICALIS EM PACIENTES ASMÁSTICOS EM SÃO LUÍS E REGIÃO METROPOLITANA.
}

\section{SENSITIZATION OF BLOMIA TROPICALIS IN ASTHMA PATIENTS IN SÃO LUÍS AND METROPOLITAN REGION.}

\begin{abstract}
Poliana Oliveira Lemos de Brito ${ }^{1}$, Thalita Diniz Duarte ${ }^{1}$, João Lucas do Carmo Lima ${ }^{1}$, Caroline Zilma Kalil de Paula Costa Pereira ${ }^{1}$; Camila Rodrigues Azevedo ${ }^{1}$, Maryangela Godinho Pereira ${ }^{2}$, Angela Falcai ${ }^{3}$.
\end{abstract}

RESUMO: As doenças alérgicas podem causar comprometimento da qualidade de vida das crianças, no qual está exposto a uma série de alérgenos, que podem gerar uma resposta do sistema imunológico como reações de hipersensibilidade. O objetivo da pesquisa foi avaliar o perfil sócioeconômico e ambiental de crianças e adolescentes sensibilizados a Blomia tropicalis (Blo t) em São Luís e região metropolitana. Aprovada pelo comitê de ética e pesquisa, com o parecer de numero: 58737916.30000.5084. Foi realizado um estudo epidemiológico por meio de análise de prontuários em pacientes do Hospital da Criança de São Luís-MA, onde foram submetidos ao teste cutâneo para o alérgeno Blo t e aplicado o questionário ISSAC e complementar. Teste estatístico utilizado foi o Qi-quadrado- Fisher. A população estudada foi constituída por 87 crianças e adolescentes, entre idade de 1 a 17 anos. Na caracterização clínica destes pacientes foi observado uma associação entre a sensibilização o Blo $t$ e a sensibilização ao Dermatophagoides pteronissynus, Dermatophagoides farinae, Periplaneta americana, Blatella germânica e ao cachorro. Observou-se também uma associação com baixa renda familiar, asma e eczema atópico. Quanto a caracterização ambiental observou-se uma correlação entre manchas na parede (mofo) e forro no quarto. Concluímos que a sensibilização o Blo t é um fator de risco para desenvolvimento de asma e eczema e esta relacionado com vários alérgenos, a baixa renda familiar e que o controle ambiental para blo t é essencial para diminuir a prevalência da asma e do eczema e para melhorar a qualidade vida.

PALAVRAS-CHAVE: asma; alérgeno; hipersensibilidade

\footnotetext{
${ }^{1}$ Aluna de Graduação em Farmácia. Aluna de Iniciação cientifica (Universidade CEUMA/ FAPEMA). E-mail: polianabrito@outlook.com

1 Aluna de Graduação em Farmácia (Universidade CEUMA).

1 Aluno de Graduação em Farmácia (Universidade CEUMA). E-mail: j.lucas_lukinhas98@hotmail.com

${ }^{1}$ Aluna de Graduação em Farmácia (Universidade CEUMA). E-mail: carol_zilma@ hotmail.com

${ }^{1}$ Aluna Graduação em Nutrição (Universidade CEUMA). E-mail: cami.azevedo@ hotmail.com

${ }^{2}$ Enfermeira. Mestranda em Saúde e Meio ambiente (Universidade CEUMA). E-mail: maryangelapereira@ hotmail.com.br

${ }^{3}$ Biomédica. Mestrado em imunologia. Doutorado em imunologia. Pós- doutorado em imunologia básica e aplicada. Pós-Doutorado em Doenças Parasitárias e Imunologia das Infecções. Professora titular da Universidade CEUMA do Programa de Mestrado em Meio Ambiente e da graduação de Farmácia e Medicina. E-mail: afalcai@gmail.com
} 
ABSTRACT: Allergic diseases can cause compromised quality of life in children, in which they are exposed to a series of allergens, which can generate an immune system response as hypersensitivity reactions. The objective of this research was to evaluate the socioeconomic and environmental profile of children and adolescents sensitized to Blomia tropicalis (Blo t) in São Luís and metropolitan region. Approved by the ethics and research committee, with the opinion number: 58737916.30000.5084. An epidemiological study was carried out by means of an analysis of medical records in patients from the Children's Hospital of São Luís-MA, where they were submitted to the skin test for Blot allergen and applied the ISSAC and complementary questionnaire. Statistical test used was the Qi-square-Fisher. The study population consisted of 87 children and adolescents, aged between 1 and 17 years. In the clinical characterization of these patients, an association was observed between the sensitization of Blot and the sensitization to Dermatophagoides pteronissynus, Dermatophagoides farinae, Periplaneta americana, Blatella germanica and to the dog. There was also an association with low family income, asthma and atopic eczema. Regarding the environmental characterization, a correlation was observed between wall spots (mold) and lining in the room. We conclude that sensitization to Blo $t$ is a risk factor for the development of asthma and eczema and is related to various allergens, low family income and that environmental control for blo is essential to reduce the prevalence of asthma and eczema and to improve the quality of life.

KEYWORDS: asthma; allergen; hypersensitivity. 


\section{INTRODUÇÃO}

As doenças alérgicas são muito comuns na infância e na adolecência. É considerado um problema mundial que geram altos custos para o sistema de saúde, absenteísmo dos pais ao trabalho e escolar das crianças, além de uma baixa qualidade de vida. As reações alérgicas podem variar de acordo com a predisposição genética e podem desencadear urticárias, eczema, conjuntivite, rinorréia, rinite, asma ou gastroenterite (Valero, Quirce et al. 2017). Estima-se que mais de 300 milhões de pessoas são acometidas pela asma e responsável por 346 mil mortes em todo mundo a cada ano. O Brasil ocupa o $8^{\circ}$ lugar mundial na prevalência da asma, com uma variação de 10 a $20 \%$ dependendo da região e da faixa etária (To, Stanojevic et al. 2012)

Algumas desta doenças alérgicas são causadas pela resposta exacerbada do organismo, chamada de Hipersensibilidade do tipo 1 ou Imediata. Dentro dos quatro tipos de hipersensibilidades, a do tipo 1 possui mecanismos fisiopatológicos diferenciados e dependentes de IgE. Este mecanismo envolve a produção de $\operatorname{IgE}$, em reposta a antígenos, que nas doenças alérgicas, denominamos de Alérgenos (Lawrence, Steinke et al. 2018). Estas reações podem ocasionar desde uma inconveniência mínima de coceiras ou até um choque anafilático, levando a morte. A reação normalmente leva de 15 a 30 minutos após a exposição ao alérgeno, embora tem alguns relatos de algumas sintomatologias terem iniciado após 10 ou 12 horas após a exposição. As doenças alérgicas resultam da interação entre a genética, exposição ambiental e a resposta imunológica que levam ao desenvolvimento e manutenção dos sintomas.

Nas alergias, os principais fatores de risco associados são os do hospedeiro, tais como a hereditariedade, gênero, obesidade e depressão e os fatores ambientais, tais como, ácaros, baratas, fungos, pólen, infecções virais, exposição ao cigarro, poluição do ar (Hallit, Assi et al. 2018). No caso dos ácaros da poeira doméstica, os principais causadores de alergia são o Dermatophagoides pteronyssinus (Der p), Dermatophagoide farinae (der f) e Blomia tropicalis (Blo t). A Blo té uma espécie mais proeminente nas regiões tropicais e subtropicais do mundo (Guilleminault and VialaGastan 2017). Nas últimas décadas, um aumento nas taxas de sensibilização para Blo $t$ tem sido reladado, e com isso um aumento no número das pesquisas. O Objetivo deste trabalho foi avaliar o perfil sócio-econômico e ambiental de crianças a adolescentes sensibilziados a Blo t em São Luís e regiões metropolitadas do Maranhão. 


\section{MATERIAL E MÉTODOS:}

Trata-se de uma pesquisa de campo realizada em setembro de 2017, no Hospital da criança de São Luís do Maranhão. Inicialmente realizou-se a seleção dos prontuários de pacientes sibilantes e asmáticos, selecionando pacientes de São Luís, Paço do Lumiar e Raposa. A população estudada foi constituída por 87 pacientes entre crianças e adolescentes.

Os pacientes foram Submetidos ao teste de hipersensibilidade cutânea (Prinkc test), para o alérgeno Blomia tropicalis e aplicado o questionário ISSAC e está dividido em três partes: a primeira sobre asma, a segunda sobre rinite alérgica e a terceira sobre eczema. Também foi realizado um questionário complementar, podendo observar respostas distintas para cada uma das diferentes questões. A este grupo determinou-se a prevalência da sensibilização ao ácaro e a correlação entre o grau de sensibilização e as características clínicas, e ambientais dos pacientes, foram avaliados os pacientes diagnosticados como portadores de asma, que assinarem o Termo de Consentimento Livre e Esclarecido (TCLE).

A reação o teste cutâneo local foi observada por 15 minutos, medindo-se os diâmetros perpendiculares da pápula, a mesma é considerada positiva se for maior ou igual $3 \mathrm{~mm}$. O controle positivo do teste foi realizado com histamina e o controle negativo, com soro fisiológico.

\section{RESULTADOS E DISCUSSÃO}

A amostra foi constituída de 87 crianças e adolescentes, sendo que a prevalência foi de $31 \%$ sensíveis a Blo t e $69 \%$ não sensíveis, com média de idade de 8,4 anos para os sensibilizados e 7,17 para os não sensibilizados. Quanto ao gênero meninos e meninas não houve diferença estatística. No entanto, houve uma diferença quanto a sensibilização a Blo t e a baixa renda familiar (Tabela 1).

Artigos científicos revelam que as doenças alérgicas estão associadas a hipótese da higiene $(\mathrm{HH})$, que mostram que a exposição alergênica no início da vida aumenta o risco de alergias, desencadeamento uma resposta imune do tipo Th2, enquanto que a exposição precoce a microrganismos com ou sem infecção, confere uma proteção, desenvolvido por uma reposta imune do tipo Th1 (Umetsu 2012, Brooks, Pearce et al. 2013, Lambrecht and Hammad 2017). E que famílias de níveis sócio-econômicos mais elevados são fatores de risco para alergia, pois quanto maior o poder aquisitivo, maiores são os cuidados higiênicos, maior é a facilidade de acesso aos recursos financeiros, favorece as brincadeiras com brinquedos eletrônicos, limitando a crianças a 
brincar dentro de casa, diminuindo as brincadeiras ao ar livre e o convívio com outras crianças (Parsons, Beach et al. 2017). Todos estes fatores contribuem para limitar o sistema imunológico. O que contradiz com o resultado encontrado nesta pesquisa que mostra a maior parte da população estudada é de baixo poder aquisitivo.

Para análises da caracterização clínica destas crianças e adolescentes evidenciou-se que a sensibilização a Blo t esta associada a sensibilização pelo Der p, Der f, pelos alérgenos de baratas Per (Periplaneta americana) e Bla g (Blatella germanica) e ao cachorro (tabela 1). A prevalência dos sintomas alérgicas dos pacientes sensibilizados a Blo t foi associado a asma e eczema atópico e não foi correlacionado com a rinite e a sibilância (tabela 1).

Como já é conhecido as doenças alérgicas são multifatoriais e entre as causas de sensibilização em pacientes com asma estão os aeroalérgenos do interior do domícilio, como o Der $\mathrm{p}$, sendo o mais frequente, Der $\mathrm{f}$, as baratas e os animais domésticos, sendo que os ácaros são mais abundantes em locais de alta umidade relativa do ar (Guilleminault and Viala-Gastan 2017, Richgels, Yamani et al. 2017, Valero, Quirce et al. 2017, Esty and Phipatanakul 2018). Estudos mostram que os testes cutâneos avaliados em $96 \%$ dos pacientes asmáticos são sensíveis a algum tipo de alérgeno, sendo a maior prevalência de positividade para extratos de alérgenos de ácaros Der p, Der f, seguida da Blo t, gato, cachorro, baratas (per a e bla g), mistura de fungos e gramínieas (Tham, Lee et al. 2016, Lok, Davis et al. 2017, Esty and Phipatanakul 2018, Wilson and PlattsMills 2018). E que esta sensibilização ocorre no início da vida com a exposição ambiental. Esta pesquisa corrobora com os trabalhos que mostram a associação da sensibilização do Blo t com a asma e o eczema atópico. 
Revista Ceuma Perspectivas, Edição Especial. V Congresso de Saúde e Bem Estar Ceuma. Vol. 30, nº2, 2017. ISSN Eletrônico: 2525-5576.

Tabela 1. Perfil sócio-econômico e clínico das crianças e adolescentes sensibilizados ou não pelo alérgeno da Blo t.

\begin{tabular}{|c|c|c|c|}
\hline Variáveis & Sensibilizados a Blo t & Não Sensibilizados a Blo t & $* \mathrm{p}$ \\
\hline No. Absoluto & $27(31 \%)$ & $60(69 \%)$ & \\
\hline idade (1 - 17) - média & 8,4 & 7,17 & \\
\hline Gênero & & & 0,5546 \\
\hline Menina & $9(33 \%)$ & $23(38 \%)$ & \\
\hline Menino & $18(66 \%)$ & $37(62 \%)$ & \\
\hline Renda familiar $* *$ & & & 0,0041 \\
\hline 1 & $1(59 \%$ & $49(82 \%)$ & \\
\hline 2 a 5 & $9(33 \%)$ & $13(22 \%)$ & \\
\hline 5 a 10 & $1(4 \%)$ & $1(14 \%)$ & \\
\hline mais de 10 & $1(4 \%)$ & $2(4 \%)$ & \\
\hline Sensibilização ao Der $\mathbf{p} * * * *$ & & & $<0,0001$ \\
\hline Positivo & $15(55 \%)$ & $17(28 \%)$ & \\
\hline Negativo & $44(44 \%)$ & $43(72 \%)$ & \\
\hline Sensibilização ao Der $\mathbf{f} * * * *$ & & & $<0,0001$ \\
\hline Positivo & $14(52 \%)$ & $13(22 \%)$ & \\
\hline Negativo & $13(48 \%)$ & $47(78 \%)$ & \\
\hline Sensibilização a Per a $* * *$ & & & 0,0006 \\
\hline Positivo & $10(37 \%)$ & $9(15 \%)$ & \\
\hline Negativo & $17(63 \%)$ & $51(85 \%)$ & \\
\hline Sensibilização a Bla g $* * * *$ & & & $<0,0001$ \\
\hline Positivo & $15(55 \%)$ & $15(25 \%)$ & \\
\hline Negativo & $12(44 \%)$ & $45(75 \%)$ & \\
\hline Sensibilizção a Gato & & & $>0,9999$ \\
\hline positivo & $1(4 \%)$ & $2(4 \%)$ & \\
\hline negativo & $26(96 \%)$ & $58(96 \%)$ & \\
\hline \multicolumn{4}{|l|}{ Sensibilização a Cachorro $* * *$} \\
\hline positivo & $3(11 \%)$ & $0(0,00 \%)$ & 0,0007 \\
\hline negativo & $24(89 \%)$ & $60(100 \%)$ & \\
\hline Sibilância & & & 0,1669 \\
\hline Sim & $20(74 \%)$ & $39(65 \%)$ & \\
\hline Não & $7(26 \%)$ & $21(35 \%)$ & \\
\hline Asma ** & & & 0,0065 \\
\hline Asmáticos & $18(66 \%)$ & $28(46 \%)$ & \\
\hline Não Asmáticos & $9(33 \%)$ & $32(53 \%)$ & \\
\hline Rinite & & & 0,1532 \\
\hline Sim & $13(48 \%)$ & $23(38 \%)$ & \\
\hline Não & $14(52 \%)$ & $37(62 \%)$ & \\
\hline Eczema Atópico * & & & 0,0307 \\
\hline Sim & $13(48 \%)$ & $20(33 \%)$ & \\
\hline Não & $14(52 \%)$ & $40(67 \%)$ & \\
\hline
\end{tabular}


Ao analisar as características ambientais das crianças e adolescentes foi encontrado uma associação com as manchas nas paredes (mofos) encontrados no quarto das crianças e a presença de forro na casa. Entretanto, não foi observado uma correlação com exposição ao cigarro, animais domésticos, dividir quarto com outras pessoas, ar condicionado no quarto, troca frequente de colchões, travesseiros e o saneamento básico (tabela 2).

O controle ambiental é importante para mnimizar a exposição aos alérgenos, sendo considerado fundamental para os tratamentos de pacientes alérgicos que apresentam asma. É uma estratégica de tratamento que pode ser mantida facilmente sem efeitos adversos, o que leva uma melhora na sintomatologia do paciente e uma melhora na qualidade de vida (Wilson and PlattsMills 2018).

Para se realizar um controle ambiental adequado é necessário requerir educação e um plano completo para diminuição da exposição alergênica no ambiente domiciliar, enquanto que medidas isoladas tem uma menor probalidade de ser eficazes. No caso da população acarínea, as medidas incluem colocação de capas impermeáveis a ácaros em colchões e travesseiros, remoção de carpetes e tapetes, limpeza de chão e móveis com pano úmido e uso frequente de aspirador de pó. Neste estudo observou-se que para Blo t a interversão seria em diminuir a umidade do ar e arejar mais o ambiente em que a criança vive. Não só o ambiente domiciliar é importante ser investigado como também outros ambientes em que estas crianças e adolescentes vivem, como por exemplo o ambiente escolar. Estudos realizados na cidade de São Paulo mostra a importância que os berçarios e creches tiveram na sensibilização aos alérgenos de ácaros (Kantor and Silverberg 2017, Ahluwalia and Matsui 2018, Kader, Kennedy et al. 2018).

Este trabalho tem um papel fundamental de mostrar a importância dos cuidados ambientais que as crianças e adolescentes sensibilizados especificamente para Blo t tem que ter para melhorar a qualidade de vida e diminuir a prevalência de asma e eczema atópica. 
Tabela 2. Perfil ambiental das crianças e adolescentes sensibilizados ou não pelo alérgeno da Blo t.

\begin{tabular}{|c|c|c|c|}
\hline Variáveis & Sensibilizados a Blo $t$ & Não Sensibilizados a Blo t & $*_{p}$ \\
\hline Exposição animais domésticos & & & 0,7553 \\
\hline Sim & $19(71 \%)$ & $43(72 \%)$ & \\
\hline Não & $8(29 \%)$ & $17(28 \%)$ & \\
\hline Mães fumantes & & & 0,0012 \\
\hline Sim & $0(0,00 \%)$ & $6(10 \%)$ & \\
\hline Não & $27(100 \%)$ & $54(90 \%)$ & \\
\hline Dividi o quarto & & & 0,2472 \\
\hline Sim & $22(82 \%)$ & $52(87 \%)$ & \\
\hline Não & $5(18 \%)$ & $8(13 \%)$ & \\
\hline Ar condicionado & & & 0,1153 \\
\hline Sim & $9(33 \%)$ & $14(23 \%)$ & \\
\hline Não & $18(66 \%)$ & $46(76 \%)$ & \\
\hline Manchas na parede (Mofo) *** & & & 0,0004 \\
\hline Sim & $18(66 \%)$ & 25 (42\%) & \\
\hline Não & $9(33 \%)$ & $35(58 \%)$ & \\
\hline Presença de Forro na casa * & & & 0,015 \\
\hline Sim & $9(33 \%)$ & $11(18 \%)$ & \\
\hline Não & $18(66 \%)$ & 49 (82\%) & \\
\hline Troca frequente de colchão & & & 0,4285 \\
\hline Sim & $8(29 \%)$ & $15(25 \%)$ & \\
\hline Não & 19 (71\%) & $45(75 \%)$ & \\
\hline Troca frequente de travesseiro & & & 0,2943 \\
\hline Sim & $10(37 \%)$ & $18(30 \%)$ & \\
\hline Não & $17(63 \%)$ & $42(70 \%)$ & \\
\hline Saneamento Básico & & & 0,2013 \\
\hline Sim & 11 (41\%) & 30 (50\%) & \\
\hline Não & $16(59 \%)$ & $30(50 \%)$ & \\
\hline
\end{tabular}

\section{CONCLUSÃO}

Presente estudo evidenciou a prevalência da sensibilização a Blo $t$ em crianças e adolescentes com asma, eczema atópico. Uma forte associação entre a sensibilização a outros alérgenos de ácaro como Der p, Der f, de baratas como Per a e Bla g e de cães. Além de estar associado ao baixo poder aquisitivo. Na caracterização do meio ambiente encontrou-se mofos e a estrutura da casa que possuía forro, sugerindo que o ambiente é úmido e abafado, com consequência no aumentando da proliferação da população acarínea. Conduto, estratégias para otimizar a redução dos níveis de alérgenos nos domicílios presisam ser avaliados na tentativa de uma melhoria na qualidade de vida. 


\section{REFERÊNCIAS}

Ahluwalia, S. K. and E. C. Matsui (2018). "Indoor Environmental Interventions for Furry Pet Allergens, Pest Allergens, and Mold: Looking to the Future." J Allergy Clin Immunol Pract 6(1): 919.

Brooks, C., N. Pearce and J. Douwes (2013). "The hygiene hypothesis in allergy and asthma: an update." Curr Opin Allergy Clin Immunol 13(1): 70-77.

Esty, B. and W. Phipatanakul (2018). "School exposure and asthma." Ann Allergy Asthma Immunol.

Guilleminault, L. and C. Viala-Gastan (2017). "[Blomia tropicalis: A house dust mite in the tropics]." Rev Mal Respir 34(8): 791-801.

Hallit, S., T. B. Assi, R. Hallit and P. Salameh (2018). "Allergic diseases, smoking, and environmental exposure among university students in Lebanon." J Asthma 55(1): 35-42.

Kader, R., K. Kennedy and J. M. Portnoy (2018). "Indoor Environmental Interventions and their Effect on Asthma Outcomes." Curr Allergy Asthma Rep 18(3): 17.

Kantor, R. and J. I. Silverberg (2017). "Environmental risk factors and their role in the management of atopic dermatitis." Expert Rev Clin Immunol 13(1): 15-26.

Lambrecht, B. N. and H. Hammad (2017). "The immunology of the allergy epidemic and the hygiene hypothesis." Nat Immunol 18(10): 1076-1083.

Lawrence, M. G., J. W. Steinke and L. Borish (2018). "Cytokine-targeting biologics for allergic diseases." Ann Allergy Asthma Immunol.

Lok, S. D., B. E. Davis and D. W. Cockcroft (2017). "Prevalence of allergen sensitization in 1000 adults in Saskatchewan." Allergy Asthma Clin Immunol 13: 9.

Parsons, M. A., J. Beach and A. Senthilselvan (2017). "Association of living in a farming environment with asthma incidence in Canadian children." J Asthma 54(3): 239-249.

Richgels, P. K., A. Yamani, C. A. Chougnet and I. P. Lewkowich (2017). "Maternal house dust mite exposure during pregnancy enhances severity of house dust mite-induced asthma in murine offspring." J Allergy Clin Immunol 140(5): 1404-1415 e1409.

Tham, E. H., A. J. Lee and H. V. Bever (2016). "Aeroallergen sensitization and allergic disease phenotypes in Asia." Asian Pac J Allergy Immunol 34(3): 181-189.

To, T., S. Stanojevic, G. Moores, A. S. Gershon, E. D. Bateman, A. A. Cruz and L. P. Boulet (2012). "Global asthma prevalence in adults: findings from the cross-sectional world health survey." BMC Public Health 12: 204.

Umetsu, D. T. (2012). "Early exposure to germs and the Hygiene Hypothesis." Cell Res 22(8): 1210-1211. 
Revista Ceuma Perspectivas, Edição Especial. V Congresso de Saúde e Bem Estar Ceuma. Vol. 30, n02, 2017. ISSN Eletrônico: 2525-5576.

Valero, A., S. Quirce, I. Davila, J. Delgado and J. Dominguez-Ortega (2017). "Allergic respiratory disease: Different allergens, different symptoms." Allergy 72(9): 1306-1316.

Wilson, J. M. and T. A. E. Platts-Mills (2018). "Home Environmental Interventions for House Dust Mite." J Allergy Clin Immunol Pract 6(1): 1-7. 